\title{
PARSEVAL'S INTEGRAL AND THE JACOBI EXPANSIONS IN SERIES OF BESSEL FUNCTIONS
}

\author{
JOHN LEKNER ${ }^{1}$
}

(Received 1 May 1984; revised 26 November 1984)

\section{Abstract}

The sums

$$
\frac{1}{M} \sum_{m=1}^{M} \cos \left\{z\left[\sin \left(\frac{m \pi}{M}\right)\right]\right\}
$$

are shown to approximate $J_{0}(z)$; the error terms are series in higher order Bessel functions, leading with $J_{2 M}(z)$. Similar sums approximate $J_{1}(z)$. These sums may be looked on as extensions of the Jacobi expansions for $\cos z$ and $\sin z$ in series of Bessel functions. They become numerically useful for $M>|z|$.

\section{Introduction}

In the theory of diffraction by a circular aperture [1], the Bessel function $J_{0}$ appears in the form of Parseval's integral [3, page 21]

$$
J_{0}(z)=\frac{1}{\pi} \int_{0}^{\pi} d \theta \cos (z \sin \theta) .
$$

I wanted a simple algorithm, based on (1), from which my second-year physics students could generate $J_{0}$ and its derivative. I tried the trapezoidal rule, which approximates (1) by

$$
S_{M}(z)=\frac{1}{M} \sum_{m=1}^{M} \cos \left(z \sin \frac{m \pi}{M}\right) .
$$

This proved to be remarkably accurate: for example $J_{0}(1)$ is given to twelve digits by the $M=6$ sum, when the trapezoidal rule is expected to give about two digits correctly (numerical aspects are discussed in the appendix).

\footnotetext{
${ }^{1}$ Physics Department, Victoria University of Wellington, Wellington, New Zealand. (C) Copyright Australian Mathematical Society 1986, Serial-fee code 0334-2700/86
} 
Why? The reason proved to lie in the Jacobi expansion [3, Section 2.22]

$$
\cos (z \sin \theta)=J_{0}(z)+2 \sum_{n=1}^{\infty} J_{2 n}(z) \cos 2 n \theta ;
$$

and to lead to extensions of Jacobi's beautiful formulae.

\section{Properties of $S_{M}(z)$}

The sum $S_{M}(z)$ approximates $J_{0}(z)$. What is the error in this approximation? We use (3) in (2) to find

$$
S_{M}(z)=J_{0}(z)+\frac{2}{M} \sum_{n=1}^{\infty} J_{2 n}(z) \sum_{m=1}^{M} \cos (2 n m \pi / M) .
$$

The sum over $m$ may be evaluated as the real part of the geometric progression

$$
\sum_{1}^{M} u^{m}=u\left(u^{M}-1\right) /(u-1), \quad u=\exp (2 n \pi i / M),
$$

and is zero unless $n=l_{M}$ where $l$ is an integer, in which case it equals $M$. Thus

$$
S_{M}(z)=J_{0}(z)+2 \sum_{l=1}^{\infty} J_{2 l M}(z) .
$$

The leading term in the power series expansion of $S_{M}(z)-J_{0}(z)$ is therefore the leading term of $2 J_{2 M}(z)$, namely $2(z / 2)^{2 M} /(2 M) ! \simeq(\pi M)^{-1 / 2}(z / 4 e M)^{2 M}$ (using Stirling's approximation). This will be small provided $|z|<M$.

For $M=1$ and 2, (5) gives series which may be obtained from (3) by setting $\theta=0$ and $\theta=\pi / 2$ (cf. [2], 9.1.46 and 47)

$$
\begin{gathered}
1=J_{0}(z)+2 \sum_{l=1}^{\infty} J_{2 l}(z), \\
\frac{1}{2}(1+\cos z)=J_{0}(z)+2 \sum_{l=1}^{\infty} J_{4 l}(z) .
\end{gathered}
$$

\section{Related sums}

If we set $\theta=\pi / 2-\phi$ in (1), Parseval's integral can be put in the form

$$
J_{0}(z)=\frac{1}{\pi} \int_{0}^{\pi} d \phi \cos (z \cos \phi) \text {. }
$$


The corresponding trapezoidal rule approximation to $J_{0}(z)$ is

$$
C_{M}(z)=\frac{1}{M} \sum_{m=1}^{\infty} \cos \left(z \cos \frac{m \pi}{M}\right) .
$$

We set $\theta=\pi / 2-\phi$ in (3) to obtain

$$
\cos (z \cos \phi)=J_{0}(z)+2 \sum_{n=1}^{\infty}(-)^{n} J_{2 n}(z) \cos 2 n \phi .
$$

On using (10) in (9) we find the companion formula to (5),

$$
C_{M}(z)=J_{0}(z)+2 \sum_{l=1}^{\infty}(-)^{I M} J_{2 l M}(z)
$$

For $M=1$ this gives a result obtainable from (10) by setting $\phi=0$ :

$$
\cos z=J_{0}(z)+2 \sum_{l=1}^{\infty}(-)^{l} J_{2 l}(z)
$$

For $M=2$ we obtain (7) again. In fact the $S_{M}$ and $C_{M}$ formulae are equivalent for even $M$. For odd $M$ they are different, and a useful result is obtained by taking their average:

$$
\frac{1}{2}\left[S_{M}(z)+C_{M}(z)\right]=J_{0}(z)+2 \sum_{L=1}^{\infty} J_{4 L M}(z) .
$$

For $M=1$ we regain (7). For $M=3$ we have

$$
J_{0}(z)=\frac{1}{6}\left\{1+\cos z+2 \cos \frac{z}{2}+2 \cos \frac{z \sqrt{3}}{2}\right\}-2 \sum_{L=1}^{\infty} J_{12 L}(z) .
$$

This approximates $J_{0}(\pi / 2)$ by $\{1+\sqrt{2}+2 \cos \pi \sqrt{3} / 4\} / 6$, correct to nine digits, and $J_{0}(\pi)$ by $\frac{1}{3} \cos \pi \sqrt{3} / 2$, correct to four digits.

The error or remainder terms in (5) and (11) have the power series expansions (obtained by using the power series expression for $J_{2 l M}(z)$ and resumming)

$$
\sum_{l=1}^{\infty}( \pm)^{l M} J_{2 l M}(z)=\sum_{N=M}^{\infty}\left(-\frac{z^{2}}{4}\right)^{N} \sum_{l=1}^{[N / M]} \frac{(\mp)^{l}}{(N-l M) !(N+l M) !}
$$

where $[N / M]$ denotes the integer part of $N / M$. On comparing these series expansions with those of $\frac{1}{2}\left(S_{M}(z)-J_{0}(z)\right)$ or $\frac{1}{2}\left(C_{M}(z)-J_{0}(z)\right)$, we obtain interesting identities. For example, for $M=3$ (and $N \geqslant 3$ )

$$
\sum_{l=1}^{[N / 3]} \frac{(-)^{l}}{(N-3 l) !(N+3 l) !}=\frac{3^{N-1}}{(2 N) !}-\frac{1}{2(N !)^{2}}
$$


and

$$
\sum_{l=1}^{[N / 3]} \frac{1}{(N-3 l) !(N+3 l) !}=\frac{1}{2}\left\{\frac{\left(4^{N}+2\right) / 3}{(2 N) !}-\frac{1}{(N !)^{2}}\right\}
$$

\section{Higher order Bessel functions}

The results of the previous sections can be extended to Bessel functions $J_{n}$ with $n>0$. We shall give results for $J_{1}$ only; formulae for $n>1$ can then be found by using the recurrence relation [3, Section 2.12]

$$
J_{n+1}(z)=\frac{2 n}{z} J_{n}(z)+J_{n-1}(z) .
$$

To find $J_{1}(z)=-J_{0}^{\prime}(z)$ we differentiate either (5) or (11). We obtain

$$
J_{1}(z)=\frac{1}{M} \sum_{m=1}^{M} \sin \left(\frac{m \pi}{M}\right) \sin \left(z \sin \frac{m \pi}{M}\right)+\sum_{l=1}^{\infty}\left[J_{2 l M-1}(z)-J_{2 l M+1}(z)\right]
$$

and

$$
J_{1}(z)=\frac{1}{M} \sum_{m=1}^{M} \cos \left(\frac{m \pi}{M}\right) \sin \left(z \cos \frac{m \pi}{M}\right)+\sum_{l=1}^{\infty}(-)^{l M}\left[J_{2 / M-1}(z)-J_{2 l M+1}(z)\right] \text {. }
$$

Setting $M=2$ in (18) or $M=1$ or 2 in (19) regains the known result [2, (9.1.48)].

$$
\sin z=2 J_{1}(z)-2 J_{3}(z)+2 J_{5}(z)-\cdots \text {. }
$$

As before, we can average (18) and (19) for odd $M$ to obtain a more accurate formula. For example, for $M=3$ we have

$$
J_{1}(z)=\frac{1}{6}\{\sqrt{3} \sin z \sqrt{3} / 2+\sin z / 2+\sin z\}+\sum_{L=1}^{\infty}\left[J_{12 L-1}(z)-J_{12 L+1}(z)\right] .
$$

The first zero of $J_{1}$, which gives the angular diameter of the first zero-intensity ring in the diffraction pattern of a circular aperture [1], can be estimated from the three terms in the curly bracket of (21) to four-digit accuracy.

\section{Discussion}

We have shown that the remarkable accuracy of certain simple trigonometric series for Bessel functions comes from the intimate relation of these series to the Jacobi formulae. A variety of extensions of the Jacobi formulae have been given, together with some consequent combinatorial identities. 
Similar expressions can be obtained for the modified Bessel functions $I_{n}$ : from the trapezoidal approximation to the integral [2, (9.6.16)]

$$
I_{0}(z)=\frac{1}{\pi} \int_{0}^{\pi} d \theta \cosh (z \cos \theta) .
$$

On using the identity (cf. (10))

$$
\cosh (z \cos \theta)=I_{0}(z)+2 \sum_{n=1}^{\infty} I_{2 n}(z) \cos 2 n \theta
$$

in the trapezoidal rule estimate for (22), we find

$$
\frac{1}{M} \sum_{1}^{M} \cosh \left(z \cos \frac{m \pi}{M}\right)=I_{0}(z)+2 \sum_{l=1}^{\infty} I_{2 l M}(z) \text {. }
$$

\section{Acknowledgement}

The paper has benefitted from helpful comments and suggestions by the referees.

\section{Appendix: Numerical aspects}

We briefly discuss some practical computational aspects of the trigonometric series approximations for $J_{n}(z)$. In the introduction we noted that the trapezoidal rule for the Parseval Integral gives (for $z=1$ and $M=6$ ) twelve digits correctly, when about two correct digits were expected. The sum in question approximates $J_{0}(z)$ by

$$
\frac{1}{6}\{1+\cos z+2 \cos z / 2+2 \cos z \sqrt{3} / 2\} \text {. }
$$

(This is the same as the $M=3$ expression of equation (14).) At $z=2$ this produces eight correct digits. In contrast, Simpson's rule with six intervals approximates $J_{0}(z)$ by

$$
\frac{1}{9}\{1+2 \cos z+4 \cos z / 2+2 \cos z \sqrt{3} / 2\},
$$

and gives four digits correctly at $z=1$, and two digits correctly at $z=2$. We thus have an interesting illustration of an integral for which Simpson's rule is much less accurate than the trapezoidal rule! (The analytical explanation is in essence contained within the body of the paper.) 
With such accuracy, one might ask whether these trigonometric sums are better than the usual power series for the evaluation of Bessel functions. The answer is a qualified yes: only with special precautions do they perform better (and then only marginally so). As an example we take the task of the repeated evaluation of $J_{0}(z)$ for $0 \leqslant z \leqslant 2$, with eight digit precision or better. The expression (A1), as its stands, is approximately $40 \%$ slower at this than a power series generated with a recursion relation, summed up to $3+[2 z]$ terms. This is because the evaluation of trigonometric functions is slow (and, to a lesser extent, so is the extraction of square roots). When (A1) is evaluated as $(c+c c+\cos (r z)) / 3$, where $c=$ $\cos (z / 2)$ and $r=\sqrt{3} / 2$ (evaluated once only), it is $7 \%$ faster than the power series. (The timings were for 200 values of $J_{0}(z), z=0.01(0.01) 2$, programmed in BASIC on an HP-85 microcomputer.) The series developed here are simple to program, but only a little faster than the power series.

\section{References}

[1] M. Born and E. Wolf, Principles of optics (Pergamon Press, Oxford, 1965).

[2] F. W. J. Olver, Chapter 9 in Handbook of mathematical functions, edited by M. Abramowitz and I. A. Stegun (N.B.S. Applied Mathematics Series No. 55, 1964).

[3] G. N. Watson, Theory of Bessel functions (Cambridge University Press, 1966). 